\title{
Application oriented connected dominating set-based cluster formation in wireless sensor networks
}

\author{
V.S. Anitha • M.P. Sebastian
}

Received: 3 February 2010 / Accepted: 21 November 2010 / Published online: 3 December 2010

(C) The Brazilian Computer Society 2010

\begin{abstract}
Clustering is a fundamental mechanism used in the design of Wireless Sensor Network (WSN) protocols. The performance of WSNs can be improved by selecting the most suitable nodes to form a stable backbone structure with guaranteed network coverage. This paper proposes a base station-controlled centralized algorithm for static sensor networks and a distributed, weighted algorithm for dynamic sensor networks. The solutions are based on a $(k, r)$-Connected Dominating Set, which is suitable for cluster-based hierarchical routing. The clusterhead redundancy parameter $k$ improves reliability, the multi-hop parameter $r$ addresses the scalability issue and the combined weight metric improves the network lifespan and reduces the number of re-affiliations. To create a stable and efficient backbone structure, the backbone sensor nodes are selected based on quality, which is a function of the residual battery power, node degree, transmission range, and mobility of the sensor nodes. Simulation experiments are conducted to evaluate the performance of both the algorithms in terms of the number of elements in the backbone structure, re-affiliation frequency, load balancing, network lifespan, and the power dissipation. The results establish the potential of these algorithms for use in WSNs.
\end{abstract}

Keywords Sensor network - Weighted clustering · Connected dominating set $\cdot$ Load balancing $\cdot$ Mobility

V.S. Anitha $(\bowtie)$

National Institute of Technology, Calicut, Kerala, India

e-mail: anithagec@yahoo.co.in

M.P. Sebastian

Indian Institute of Management Kozhikode, Kozhikode, Kerala, India

e-mail: sebasmp@iimk.ac.in

\section{Introduction}

Development of wireless sensor networks (WSN) is enabled with the recent technological advancement in wireless communications, low-power consumption processors, and highly integrated digital electronics. A dense sensor network consists of hundreds or thousands of sensor nodes with capabilities of sensing, processing, and communicating and are deployed in uncontrolled hostile environment and for unattended operations. A WSN typically has little or no infrastructure. The two approaches to deploy sensors in a WSN are the deterministic deployment and the random deployment [24]. In deterministic deployment, sensors are placed exactly at pre-engineered positions, whereas in random deployment, large numbers of sensor nodes are dispersed randomly by dropping from a plane or throwing from a moving vehicle. Failures are inevitable in WSNs as the battery-drained nodes create holes in the network topology, causing connectivity and information loss. Therefore, use of energy-aware algorithms with failure detection and maintenance mechanism is crucial in the design of WSNs.

A wireless system is expected to support 'anytime anywhere' type of service and this characteristic makes it a very attractive technology. A cellular telephone system provides a connection to the Public Switched Telephone Network (PSTN) for any user within the radio range of the system. The available bandwidth of cellular radio systems is low and it allow voice and data communication through handheld phones. Bluetooth allows users to make ad hoc wireless connections between devices like mobile phones, desktop, or notebook computers without any cable. It supports uni-cast and multi-cast connections and uses the concept of master and slave. A device needs to wait until the master allows it to talk.

Ad hoc networks are wireless, infrastructure less, multihop, and dynamic networks established by a collection of 
mobile nodes. Among the existing wireless ad hoc network models, Mobile Ad hoc Networks (MANETs) are closely related to WSNs. They share some common characteristics, such as wireless communication media, the network topology and, bandwidth and energy constraints. Hence, many existing concepts, protocols and techniques used in cellular, Bluetooth, MANET, and traditional wireless networks are applicable to WSNs, also. But some unique features and applications of WSNs make them different from the other wireless networks. The unique characteristics of a WSN include data-centric nature of the network, physical resource constraints, environment-driven nature, and the correlated data problem. Energy conservation is a more relevant matter than capacity for most of the sensor network applications [10]. Also, in generic, the WSN nodes are deployed densely than in MANETs. These features together with the wide ranges of application requirements necessitate the design of new or modified protocols for WSNs.

The Sensor network applications include, but are not limited to (i) military and civilian applications (e.g., battle field surveillance, sensing of intruders, reconnaissance, and targeting systems); (ii) academic, industrial, and home applications [22, 26] (e.g., automotive manufacturing, monitoring product quality, managing inventory, and home automation); (iii) physical world applications [1, 8, 9, 20, 23] (e.g., environmental monitoring like early fire detection in forests, detection, and monitoring of environmental changes in oceans and plains, chemical, or biological detection and flood detection); and (iv) health applications [28, 33] (e.g., monitoring and assisting of patients and drug administration). Most WSN applications require scalability, load balancing, and prolonged network lifetime. The use of networked system of sensors can limit the chance for personal interaction in the usually dangerous reconnaissance missions [3].

\section{Background}

The concept of virtual backbone infrastructure is used by researchers for broadcasting, area monitoring and process scheduling. Keeping a battery operated sensor node always active is not desirable as it quickly depletes its energy resources. The lifetime of a sensor node can be extended if it uses energy only during the specified time slot allotted to it. Thus, every sensor node must turn off its circuitry to conserve energy when its time slot has passed. The concept of a backbone structure to the sensor network allows some of the nodes to be off when not in use. This saves power and prolongs the network lifetime. However, to support the communication efficiently, the members of the backbone need to be always active and stable. Hence, the selection of the backbone members must be done judiciously.

A Connected Dominating Set (CDS) serves as a virtual backbone for WSNs to reduce the routing overhead [27].
A Dominating Set, DS, is defined as a set of nodes in the network such that all nodes in the network which are not in the DS are adjacent to at least one node in the DS. When the graph induced by a DS is connected, then it is called a CDS. For any arbitrary network, the problem of computing the CDS of minimum cardinality is an NP-complete problem [18].

CDS-based clustering of sensor nodes into groups allow sensors communicate information to the Sink through the stable backbone nodes. This saves considerable amount of energy and helps in prolonging the network lifespan. Other than to support network scalability and power efficiency, clustering conserves communication bandwidth, and stabilizes the network topology. The topology of a sensor network influences its latency, robustness, and data aggregation capability. Flooding for broadcasting purpose can cause broadcast storms. Therefore, recent broadcasting, activity scheduling, and area monitoring algorithms use the concept of virtual network infrastructure known as backbone structure (B-structure). The construction of a stable B-structure offers better support for efficient communication. The backbone is a subset of the sensors that perform data communications and serves the nodes that are not part of the backbone [29]. A previous research work [30] has shown that the backbone reduces the routing overhead dramatically. A vast literature about the backbone construction can be found in [31]. Cluster-based routing is a special case of backbonebased routing with the nodes in the backbone considered to be clusterheads [14].

This paper proposes centralized and distributed solutions for creating and maintaining B-structures for static and dynamic WSNs, respectively. These algorithms are based on a $(k, r)-C D S$ and the nodes for the construction of the B-structure are elected based on the residual battery power, node degree, transmission range, and mobility. To provide redundancy, the CDS is constructed in such a way that any sensor node is covered by at least $k$ backbone nodes within the $r$-hop distance. The proposed clustering schemes enable the formation of stable clusters and use techniques to maintain the cluster structure as stable as possible with minimal control messages. To adhere to the cluster-based approach, after constructing the virtual backbone with CDS members, clusters are created with CDS members as clusterheads. Even though our proposals avoid the necessity for network wide synchronization, clusterheads allow its members to switch to the low power sleep mode through the scheduling mechanism. This scheduling is in the round-robin order. Duty-cycling is achieved by selecting only a subset of the nodes in WSN (the CDS members, in this case), to remain active for communication and control.

The centralized algorithm assumes a known topology and creates a B-structure with minimal number of members in the backbone. Here, the control overhead is relatively high 
for learning the network topology and grows with the network size. Hence, it is suitable for static and known topology networks. The distributed algorithm creates stable, scalable, and load balanced B-structure, which can be used for large and dynamic topologies.

The rest of the paper is organized as follows. Section 2 reviews the previous works while Sect. 3 describes the system model and the performance parameters. Section 4 presents the design of the proposed protocols. Section 5 evaluates the performance of the protocols, and Sect. 6 concludes the paper.

\section{Related work}

A sensor network can be considered as an embedded system which has self-configuration, self-healing, multi-hop communication, and dynamic routing. A survey on the design issues, applications, and techniques for WSNs is presented in [4]. Routing in sensor networks got lot of attention in the recent years. A survey on the routing issues and techniques in WSNs is presented in [2]. Grouping of sensor nodes into clusters has been studied extensively for achieving network scalability, energy efficiency, and stability. Low energy consumption is a critical design requirement for WSN applications. Efficient routing techniques play a major role in the energy consumption and maximize the lifetime of a sensor network.

Low Energy Adaptive Clustering Hierarchy (LEACH) [21] is one of the most popular clustering algorithms for WSNs. It creates clusters based on the received signal strength and uses clusterhead nodes to route packets to the Sink. The role of the clusterhead is rotated periodically among the nodes of the cluster in order to balance the load. The clusterhead election normally does not take care of the capability of a node to act as clusterhead, and hence a node with low residual battery power may be selected as clusterhead. Also, such elections cannot be applied to large sensor networks. Power Efficient Gathering in Sensor Information Systems (PEGASIS) [25] is a greedy algorithm and is power efficient. However, it does not scale well and is not applicable to sensor networks with unknown topologies.

Hybrid Energy Efficient Distributed Clustering (HEED) [37] is an energy efficient, hybrid, and distributed clustering algorithm. It periodically selects a clusterhead based on the residual energy and communication costs. It needs multiple broadcasting for cluster formation, thus consumes more energy. Distributed Weight-based Energy Efficient Hierarchical Clustering [16] proceeds in a distributed manner. It aims at generating balanced cluster sizes and needs significantly low energy consumption in inter and intra-communication than HEED. The connected dominating set concept was first introduced by Guha and Kuller [19]. They have proposed three centralized, greedy approximation algorithms. The algorithm proposed by Das et al. [15] first computes the dominating sets and then additional nodes are added to covert the DS into connected DS. The algorithm proposed by $\mathrm{Wu}$ and $\mathrm{Li}$ [34] first constructs a connected dominating set and then deletes the redundant nodes based on some rules. The performance ratio is not given with the proposal. Wan et al. [32] proposal requires the construction of a spanning tree to select the dominating nodes. Distributed Solutions for computing approximations to the minimum CDS problem have been proposed in [12] and [13]. To improve power efficiency, stability, and network lifetime, along with the reduction in the total number of dominating nodes, the quality of these dominating nodes is also to be considered.

Some approximation algorithms have been proposed to construct the $k$-connected $m$-dominating set $(\mathrm{km} \mathrm{CDS}$ ) to improve the fault tolerance and routing flexibility. The protocol [36], first constructs the $m$-dominating set and then makes it to $k$-connected by augmenting the first. The message complexity of this approach is very high. Wu and $\mathrm{Li}$ [35] proposed a distributed algorithm with low message complexity to construct a $\mathrm{kmCDS}$ for generic values of $k$ and $m$. This approach requires a high degree of redundancy for the network topology. The major design objective of the dominating set-based/Connected Dominating set-based approach is the construction of a virtual backbone with minimum number of nodes (the quality of a node to act as a backbone member is not at all considered). As a solution to this problem, a centralized, scenario-based, weighted, and dominating set-based algorithm for mobile ad hoc networks is proposed by the authors in [5]. In [6], the authors have proposed a distributed, adaptive, and weighted clustering algorithm for mobile ad hoc networks.

The Weighted Clustering Algorithm (WCA) [11] is a combined weight metric-based clustering approach to form single-hop clusters. In WCA, a node with minimum sum of four indices is elected as the clusterhead. To determine a clusterhead, the algorithm considers the ideal number of nodes that a clusterhead can handle, the sum of distances to other nodes in its radio distance, mobility, and the time it becoming the clusterhead. WCA has improved performance than the other clustering algorithms as it considers many weight factors. All nodes in the network need to compute their weights and should know the weights of other nodes before starting the clustering process. This process takes more time and bandwidth, and delays the cluster formation. Moreover, limiting the cluster dimension to a single-hop reduces the scalability in a large scale networking environment.

From the discussion above, it is observed that the protocols proposed in the literature consider different design factors to suit to certain type of applications for a particular topology. A generic multi-purpose protocol which is stable, 
scalable, adaptive, and power efficient with load balancing is not available in the literature. So, in this paper, we propose the design of an adaptive (multi-purpose) clustering protocol, that is scalable, stable, adaptable, power efficient, and allows load balancing.

\section{The WSN model}

In this section, we present the WSN model and the design parameters of centralized and distributed scenario-based clustering algorithms.

\subsection{The network model}

The basic characteristics of any sensor network include dense deployment of sensor nodes, self-organizing capabilities, constraints of power and other resources, dynamic network topology, mobility of nodes, node failure, multi-hop routing, and short-range broadcast communications. Sensors are deployed in an ad hoc manner in remote or hazardous location without the need for any infrastructure to monitor events and gather data about the environment. The nodes may be static or mobile, homogeneous, or heterogeneous with different energy levels or transmission range.

The sensor network is modeled using a graph $G=$ $\left(V, E_{t}\right)$, where $V$ represents a set of wireless sensor nodes and $E_{t}$ represents the connectivity set. If $u, v \in V$ then $(u, v)$ $\in E_{t}$ if and only if $u$ and $v$ are within the transmission range of each other. The Dominating Set (DS) of graph $G$ is a subset $M \subseteq V$, such that each node in $V$ not in $M$ is adjacent to some node in $M$ [7]. A connected dominating set (CDS) for a given graph $G$ is a dominating set $N$ with the additional requirement that the nodes in $N$ induce a connected subgraph of $G$.

The $(k, r)$-Dominating Set of graph $G$ is defined as a subset $D \subseteq V$, such that each node in $V$ not in $D$ is at a distance less than or equal to $r$ from at least $k$ vertices in $D$. The $(k, r)-C D S, C$, is a $(k, r)-D S$ of $G$ which induces a connected sub-graph of $G$.

Clustering can be modeled as a graph-partitioning problem. The centralized solution for finding $(k, r)-C D S$ can be used if all nodes in the network know the topology of the entire network. Distributed solutions are suitable for synchronous and asynchronous networks. Nodes in the CDS form the backbone of the sensor network.

\subsection{Benefits of $(k, r)$-CDS-based clustering}

The efficiency of multi-cast or broadcast routing can be improved through the use of a CDS as backbone. A CDSbackbone eliminates the redundant broadcasts and saves power. It balances the network load and increases the number of nodes in sleep mode. Restricting the routing through the CDS results in a significant reduction in message overhead associated with routing updates. Reduction in the number of active nodes reduces the end-to-end delay between the Sink and sensor nodes. Most of the CDS-based protocols try to reduce the total number of elements in the backbone structure. This reduction, in turn, reduces the control overhead and the size of the route table. As the backbone nodes coordinate the neighboring ordinary nodes, the increase in the number of ordinary nodes associated with a backbone node increases its overhead. This overload in the backbone causes quick depletion of the residual battery power. Thus, providing clusterhead redundancy for each node in the network improves the stability of the backbone. In a CDS based protocol, too much redundancy results in more control overheads and too little redundancy adversely affects the connectivity. The capability of a node to become a clusterhead mainly depends on factors like residual battery power, node degree, transmission range and mobility.

Fault tolerance, reliability, and routing flexibility are necessary for efficient routing. Nodes in the WSNs are more prone to failures which may have mobility and with dense population. Thus, it is important to maintain certain degree of redundancy in a CDS. The proposed algorithms use the clusterhead redundancy parameter $k$ to improve reliability, and the multi-hop parameter $r$ to address the scalability issues.

\subsection{Design parameters}

Some characteristics of a node can directly or indirectly influence the efficiency and the rate of control of messages generated by the associated clustering protocol. The properties or metrics, we consider are connectivity, residual battery power, transmission range, and the local stability of the node. These metrics determine the adequacy of nodes to act as clusterheads. In the proposed algorithm, CDS member election is based on a combined weight-metric mechanism. The node weight is the weighted linear combination of the above mentioned metrics, and the weight factors are dependent on the service type being deployed in the network. The weight metric can also be an attribute of a single property, but a generalized vision is preferred as ad hoc networks exist in different scenarios and that clustering can be based on different priority properties largely depending on the communication scenarios and environment. Different services have different requirements. Depending on the service type, the most important parameters from the service view point are to be considered with high parameter weights, which results in the best suited CDS selection. The node with the highest weight in its neighborhood can be chosen as a member of CDS.

The position of the clusterhead is relevant in the clusterbased approach. The degree of a node is high if it has more 
number of nodes within its transmission range. This parameter helps to reduce the cardinality of the CDS. A node with high value of transmission power covers a large area, and hence this parameter helps in reducing the total number of clusters in the WSN. Since the clusterheads play the leading role in the communication process, their energy consumption is more in comparison with the ordinary nodes. Therefore, a node with sufficient battery power is to be selected as clusterhead to reduce the amount of overhead incurred due to clusterhead re-election and to avoid nodes dropping out of the network prematurely. Certain clustering scenarios demand clustering based on the mobility of nodes, and hence the formation of clusters is determined by the mobility pattern of nodes to ensure maximum cluster stability.

The processing power, memory capacity, bandwidth, link quality or availability, and transmission rate are also possible parameters which can be considered for the selection of a clusterhead. However, these factors are more relevant to MANETs than for WSNs because typical applications of MANETs include non-real time data, multimedia, and voice communication. The typical WSN-applications require transmission of few bytes periodically or upon request or according to some external event. We now discuss the design parameters in detail.

Node degree Each node computes its degree. The degree of a node $v$ is the total number of nodes within the transmission range of $v$ and is computed as

$D g_{v}=\sum_{u \in V, u \neq v}\left\{D_{u v}<T_{x}\right\}$,

where $D_{u v}$ is the distance between node $u$ and $v$ and $T_{x}$ is the transmission range.

Residual battery power A node with high residual battery power, $B_{v}$, can perform as clusterhead for a longer duration. Hence, computing the residual battery power is a better option than the consumed battery power or the cumulative time during which the node acts as the clusterhead.

Transmission range The transmission range, $T_{r}$, of a node is a factor that determines the quality of the clusterhead. This parameter is more relevant in the case of heterogeneous networks.

The combined weight $W_{v}$ for each node $v \in V$ is calculated as

$W_{v}=W_{1} * b_{v}+W_{2} * d g_{v}+W_{3} * t_{r}$,

where $W_{1}, W_{2}$, and $W_{3}$ are the weight factors for the corresponding parameters and $b_{v}, d g_{v}$, and $t_{r}$ are normalized values of $B_{v}, D g_{v}$, and $T_{r}$, respectively.
Mobility Most of the network architectures assume that the sensor networks are stationary which need not be true. For instance, in a target detecting or tracking application, it is deemed necessary to support mobility of sensor nodes. Stability of a node denotes how stable the node is for being a clusterhead. To compute mobility, each node in the dominating set needs to find out the distance from its neighboring nodes. For distance computation, Friis [17] free space propagation model is used.

The received power, $P_{r}$ is computed as

$P_{r}=P_{t} * G_{t} * G_{r} * \frac{\lambda^{2}}{(4 * \pi * D)^{2}}$,

where $P_{r}$ is the power received by the receiving antenna, $P_{t}$ is the power input to the transmitting antenna, $G_{t}$ and $G_{r}$ are the gains of transmitting and receiving antennas, respectively, with $\lambda$ as the wavelength, and $D$ as the distance. $P_{r}$ is inversely proportional to the square of the distance.

Instead of finding the exact physical location, an approximate distance at time $t$ between nodes $v$ and $u$ is calculated using (3) as

$D_{t}^{v, u}=\frac{k}{\sqrt{P_{r}}}$,

where $v \in$ dominating set $D S, u$ is an element of the set of neighboring nodes of $v$ and $k$ is a constant. $D_{t}^{v, u}$ is the distance between $v$ and $u$ at time $t$. The node mobility significantly influences the network performance.

Relative mobility between $v$ and $u$ indicates whether they are coming closer to or moving away from each other. The relative mobility of node $u$ with respect to node $v$ at time $t$ is given by

$R M_{t}^{v, u}=D_{t}^{v, u}-D_{t-1}^{v, u}$,

$R M_{t}^{v, u}$ is positive if node $u$ is moving away from $v$ and negative if $u$ is coming closer to $v$. The distance from $v$ to $u$ is measured at certain time interval for $T$ times, and thus $R M_{1}^{v, u}, R M_{2}^{v, u}, \ldots, R M_{T}^{v, u}$ are calculated. The standard deviation of relative mobility gives the variation of the distances over a time period, $T$ as

$S D R M=\sqrt{\frac{1}{T} \sum_{i=1}^{T}\left(R M_{i}-\overline{R M}\right)^{2}}$,

where

$\overline{R M}=\frac{1}{T}\left(R M_{1}^{v, u}+R M_{2}^{v, u}+\cdots+R M_{T}^{v, u}\right)$.

The local stability (LSTAB) of a node $v \in$ dominating set $D S$ with respect to all its neighbors is the mean of standard deviation of the relative mobilities of all its neighboring nodes. 
A low value of this is an indication of a stable node. This stability is either due to less mobility or due to group mobility (the node $v$ and all its neighboring nodes move in the same direction with more or less same velocity). lstab is the normalized value of $L S T A B$.

The quality of a backbone node, $v$, is calculated as

$Q_{v}=W_{v}+W_{4} * l s t a b_{v}$,

where $W_{4}$ is the weight factor associated with the local stability. Suitable values are assigned to $W_{1}, W_{2}, W_{3}$, and $W_{4}$ based on the required application such that $W_{1}+W_{2}+$ $W_{3}+W_{4}=1$. The parameters considered may not contribute equally to the quality computation (different weights can thus be given to different parameters based on the scenario). For example, if the network is more or less static, then the local stability computation can be eliminated or can assign a very small value to $W_{4}$. It is possible to consider more than four parameters if found relevant.

\section{The proposed protocols}

In a cluster-based approach, the re-election of the clusterhead and the frequent information exchange among the participating nodes incur high computation and communication overheads. This is minimized by preserving the cluster structure as stable as possible.

\subsection{The centralized connected dominating set-based weighted and adaptive clustering algorithm for sensor networks (CCWACS)}

The proposed algorithm assumes that the sensor nodes are stationary and uses a greedy heuristic for the computation of $(k, r)$-CDS. This mechanism ensures that all sensor nodes in the network other than the nodes in the backbone are covered by at least $k$ elements in the backbone within the $r$-hop distance. The two phases of CCWACS are Clustering setup (meant for backbone creation) and Cluster formation.

\subsubsection{Clustering setup phase}

The Clustering setup phase computes $(k, r)$-CDS based on the individual node weight heuristics. The nodes in $(k, r)$ $C D S$ act as the B-structure members. The weight heuristic uses some parameters to find the suitability of the nodes acting as the backbone element. The backbone members are elected on the basis of the node degree, the residual battery power and the transmission range. All nodes in the $(k, r)$-CDS need not act as clusterheads and those which are not acting as clusterheads act as gateway nodes. The second phase is the Cluster formation phase where each regular node selects the most suitable node from the backbone as its clusterhead. From among the $k$ clusterheads, each node selects only one node from the backbone structure as its clusterhead based on its quality, computed using (7). A node in $(k, r)-C D S$ without any cluster members attached acts as a gateway. The node mobility is not considered in the Clustering setup phase because it requires more control messages to compute the local stability of all nodes in the network with respect to their neighbors. But the local stability of the backbone member node is considered in the Cluster formation phase. Hence, only the nodes in the B-structure need to compute the local stability. The algorithm for computing the $(k, r)-C D S$ is as follows:

1. Initialize, the status of all nodes in the network as Ordinary.

2. Compute the weight of each node based on the connectivity (hello messages are used for this purpose), transmission range and the residual battery power. The node, $v$, with the highest weight is selected as a member of the backbone structure and the status of that node is assigned as ClusterHead. Use Node ID for tie breaking.

3. Assign the status of all 1-hop neighboring nodes of $v$ as NominatedCh and modify the relevant fields (i.e., NodeID, Number of nodes within the $r$-hop distance, weight, number of clusterheads within the $r$-hop distance, status) of all nodes within the $r$-hop distance of $v$.

4. Elect the highest weighted, uncovered node with NominatedCh as status and assign its status as ClusterHead. The Node ID is used for tie breaking.

5. If all nodes with NominatedCh as status are covered then a node with the highest number of Ordinary nodes as neighbors is selected as ClusterHead. Use the highest weight for tie breaking.

6. The process continues until all nodes (other than the ClusterHead nodes) have been covered by at least $k$ ClusterHead nodes within the $r$-hop distance.

The centralized algorithm for $(k, r)$-CDS computation is as shown in Fig. 4. The second phase involves cluster formation and management which is common for both central and distributed algorithms and the cluster formation and management issues are discussed in Sect. 6.

\subsubsection{Example}

Figure 2 shows the computation of a $(2,2)-C D S$ for the network topology shown in Fig. 1. Here, the clusterhead nodes which form the backbone are marked with a black spot. Three possible status levels for a node are ClusterHead, NominatedCh and Ordinary node (C, N and O). Initially, all nodes are assigned the status as Ordinary. In Fig. 1, the quadruple $[w, x, y, z]$ represents [Number of neighbors within the $r$-hop distance, Weight, Number of backbone elements within the $r$-hop distance, status]. The node with 
maximum weight is selected as the ClusterHead. If there are more nodes with the same weight, tie breaking is done by choosing the lowest-ID. Node G has the highest weight and is thus selected as the clusterhead. All neighboring nodes of $\mathrm{G}$ are assigned the status of NominatedCh and all the nodes within the $r$-hop distance are accounted for the selected ClusterHead as in Fig. 2[A].

Node $\mathrm{G}$ has four adjacent nodes (E, F, J, and $\mathrm{K}$ ) and nine nodes at 2-hop distance. Nodes E, F, J, and K are assigned with status NominatedCh and the third field of all these

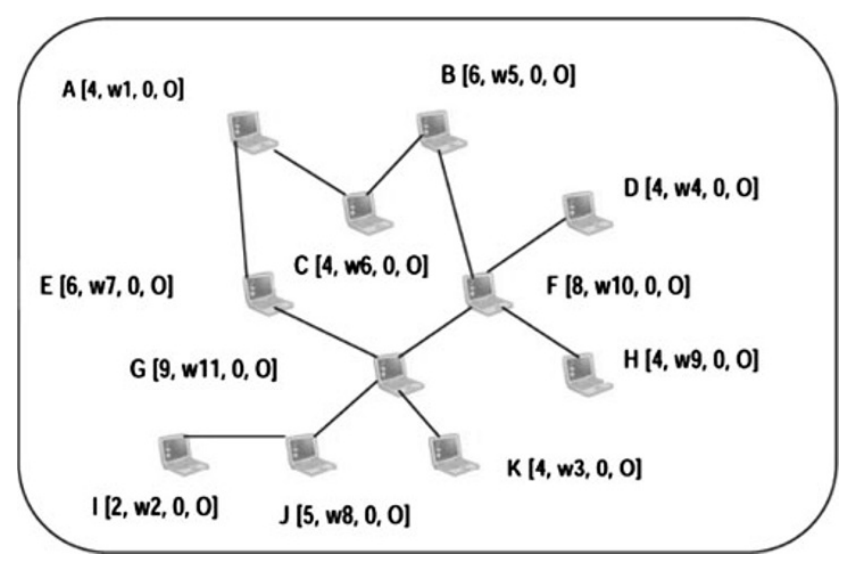

Fig. 1 A typical sensor network nodes is modified accordingly. Among the NominatedCH nodes, node F has the highest weight. Since F is not covered, it is selected as the next node in the backbone and its status is assigned as ClusterHead. All its neighbors are assigned the status NominatedCh and the relevant field of the $r$-hop neighbors is also updated to reflect the new selection as shown in Fig. 2[B]. To select the next backbone member, the algorithm searches for an uncovered node with status NominatedCh. But in this example, all NominatedCh nodes are covered, and hence a NominatedCh node with highest Ordinary neighbor nodes is to be selected. Here, node B, E, and $\mathrm{J}$ have same number of Ordinary neighbor nodes. But node $\mathrm{J}$ is selected based on its weight (Fig. 2[C]). During the next iteration, node $\mathrm{E}$ is added to the backbone structure (Fig. 2[D]). The algorithm terminates when all the nodes are covered.

\subsubsection{Analysis}

We now show that the centralized algorithm computes $(k, r)-C D S$ for any connected static network. A wireless sensor network is modeled as a graph $G=\left(V, E_{t}\right)$, where $V$ represents a set of wireless sensor nodes and $E_{t}$ represents the connectivity set. A link exists between two vertices $u$ and $v$ if the Euclidean distance between them is within their transmission range. A connected Dominating Set CDS is a

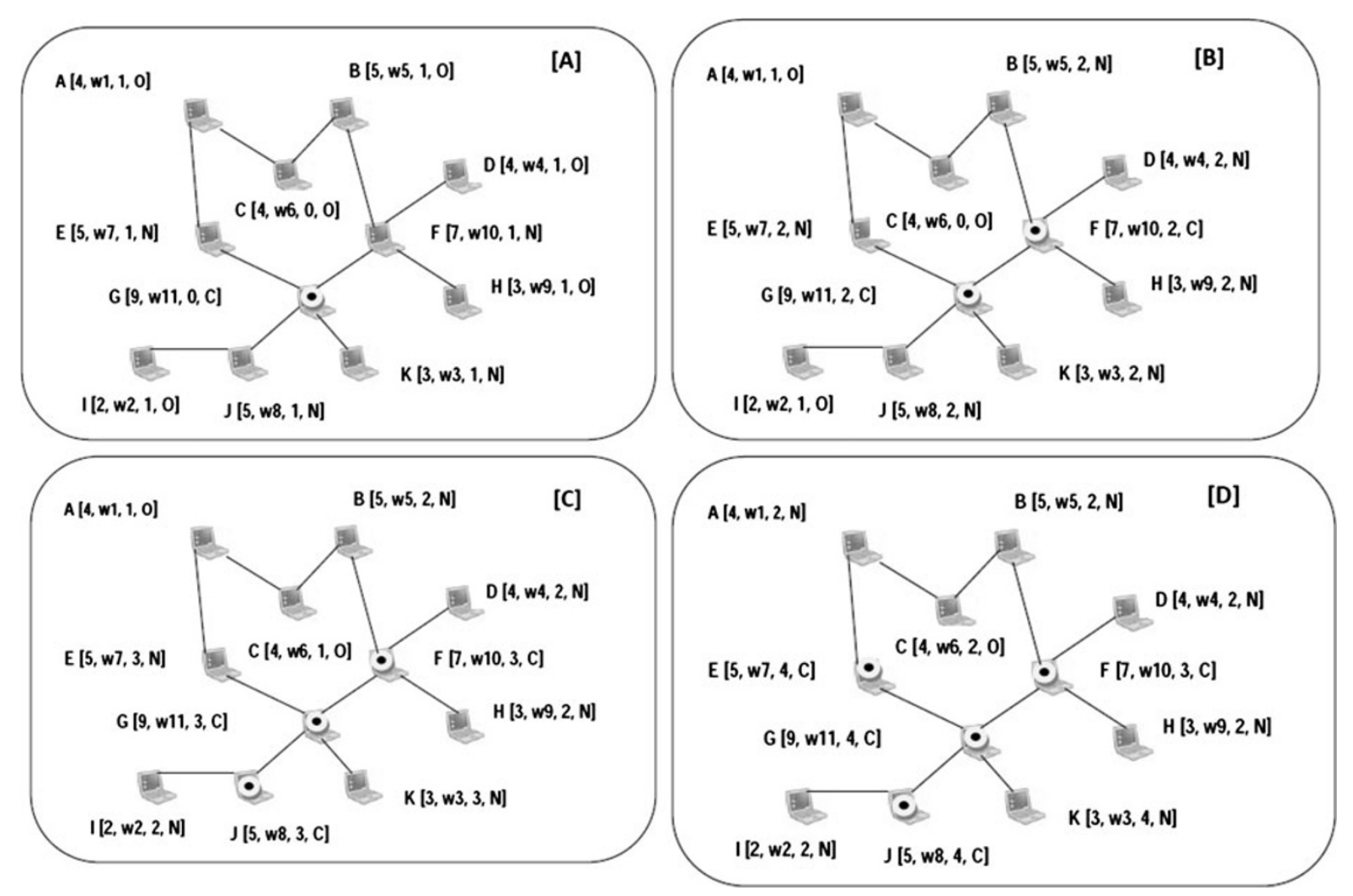

Fig. 2 Centralized computation of (2, 2)-CDS 


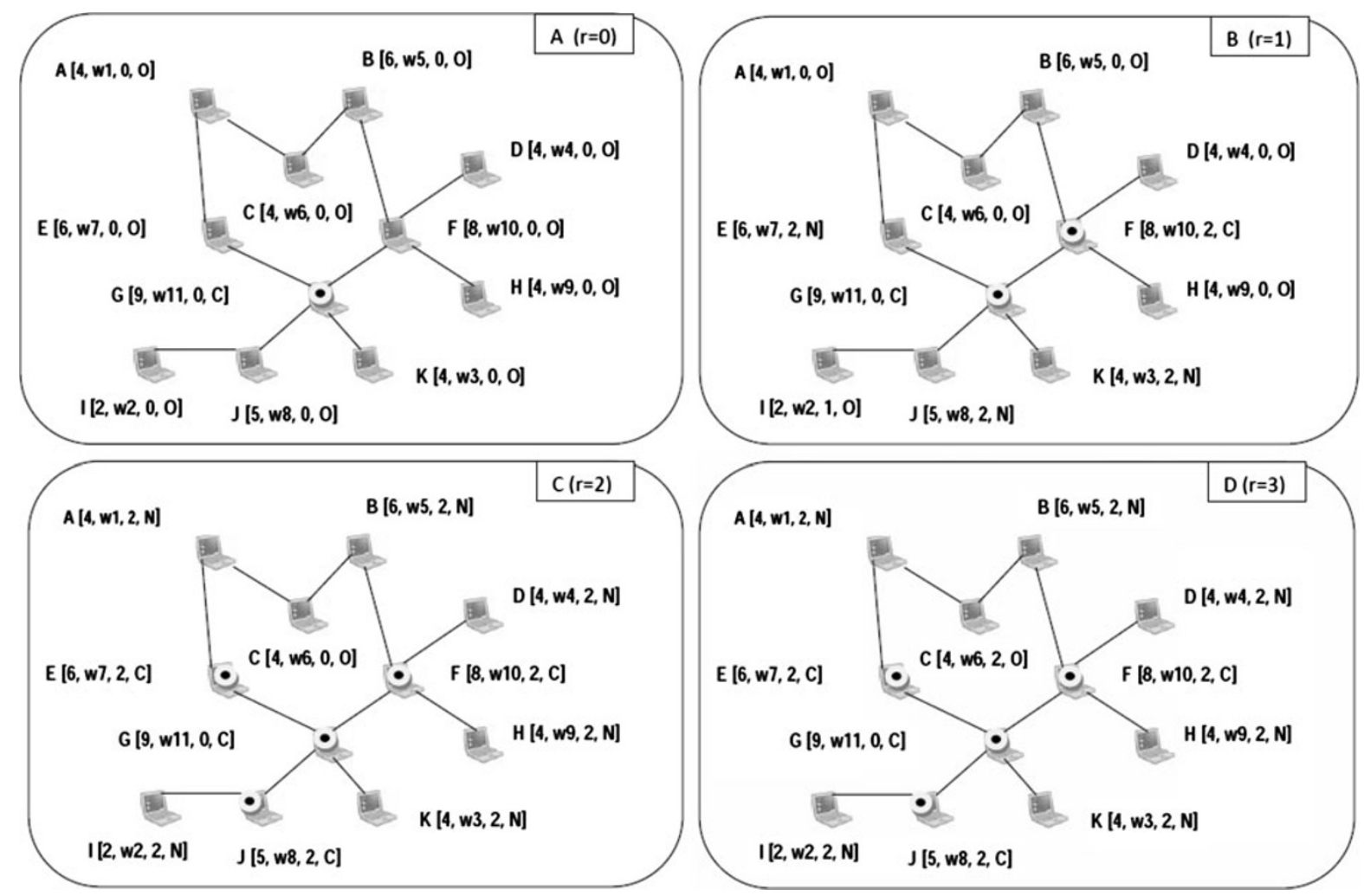

Fig. 3 Distributed computation of $(2,2)-C D S$

connected subset of $V$, if all nodes in $V-D$ are within the $r$-hop distance of at least $k$ elements in CDS.

Theorem 1 The $(k, r)$-CDS algorithm correctly computes the $(k, r)$-CDS for any arbitrary connected graph.

Proof Let the network be with $n$ number of nodes. All these nodes belong to the set $D_{r}^{0}$, where $D_{r}^{i}$ means the set of nodes covered by at least $i$ members in the connected dominating set $C$, within the $r$-hop distance. Initially, $C$ is empty and the elements are added one by one iteratively. The first element in $C$ is the node with the highest weight. The addition of a new member in $C$ moves one or more members from $D_{r}^{i}$ to $D_{r}^{i+1}$. In case of tie, the node with the lowest NodeID succeeds. In each iteration, a new node is added to the set $C$. The uncovered node $v$, which is a neighbor of any node in $C$, with the highest weight, is selected in each iteration and $v$ is added to the set $C$. In the absence of any uncovered NominatedCh nodes, the algorithm selects the NominatedCh node with the highest number of Ordinary nodes as neighbors. Since $v$ is augmented to a connected component, $C$ is also connected.

Termination: The total number of nodes in the network is $n$ which is finite. The algorithm terminates when all the nodes except the members in the backbone are covered by at least $k$ nodes in the ClusterHead node set within the $r$-hop distance or all nodes belong to the ClusterHead set. Hence, the total number of iterations is at most $n$ and the algorithm terminates within a finite amount of time.

5.2 The distributed connected dominating set-based, weighted, and adaptive clustering algorithm for sensor networks (DCWACS)

The algorithm assumes that the sensor network is with dynamic topology. So, DCWACS does not seek the information about the whole network topology. The nodes need to know only their $r$-hop neighborhood. The $(k, r)-C D S$ computation is done distributively. The elements in the $(k, r)$ $C D S$ act as backbone. The distributed algorithm works in two phases, the first phase is the Location learning and the second phase is the CDS-computation.

\subsubsection{Location learning}

We assume that there is only one Sink (Base Station) and this phase stems from the Sink. In the absence of a Sink, any node in the network with sufficient residual battery power is selected at random to act as the Sink. This phase involves collection of the quality of $r$-hop neighborhood and computation of the distance of each node from the Sink.

The Location learning algorithm is as shown in Fig. 5. For learning the weight of the $r$-hop neighborhood, each 
Fig. 4 Centralized algorithm for $(k, r)-C D S$ computation

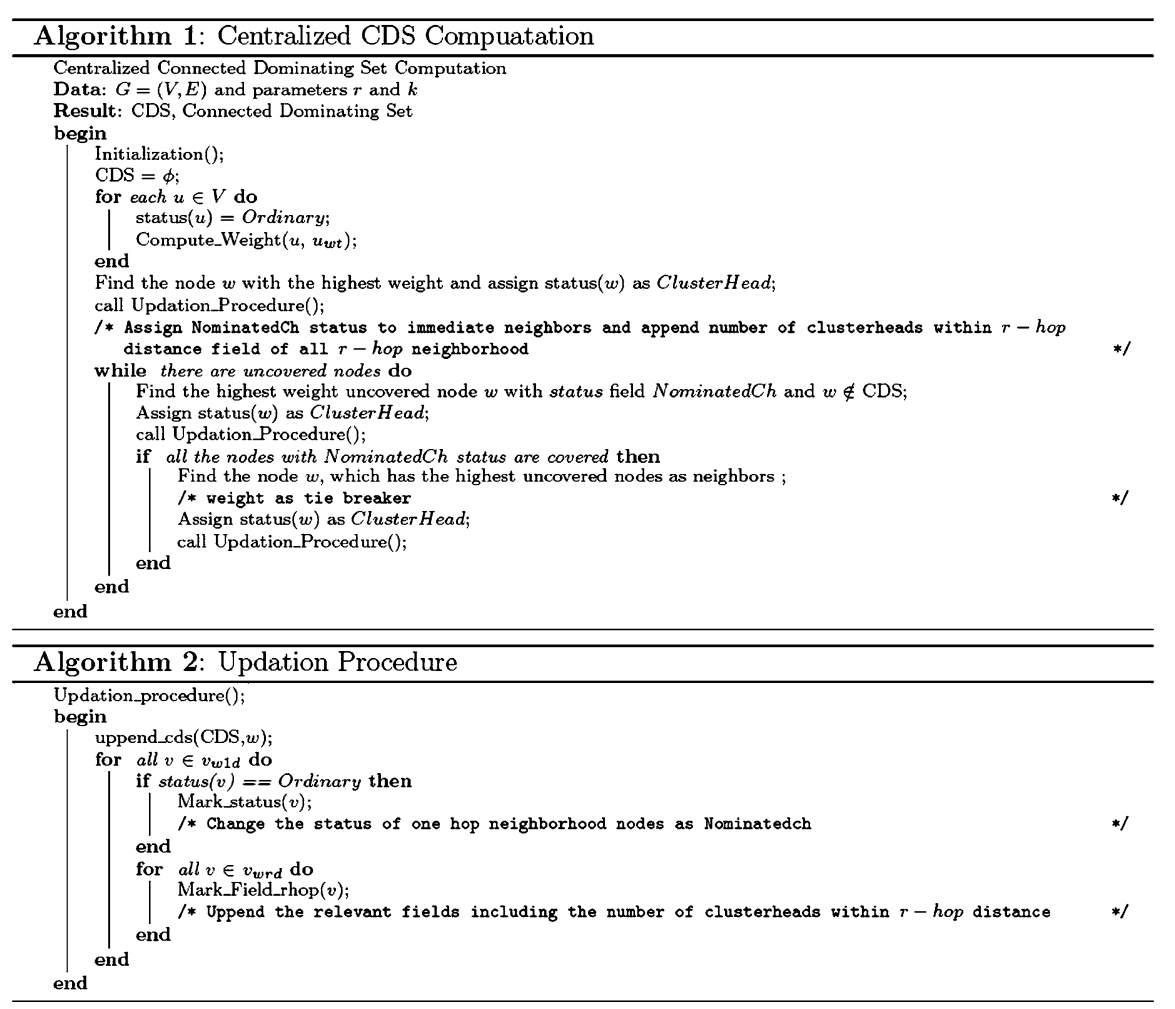

Fig. 5 Location learning phase DCWACS

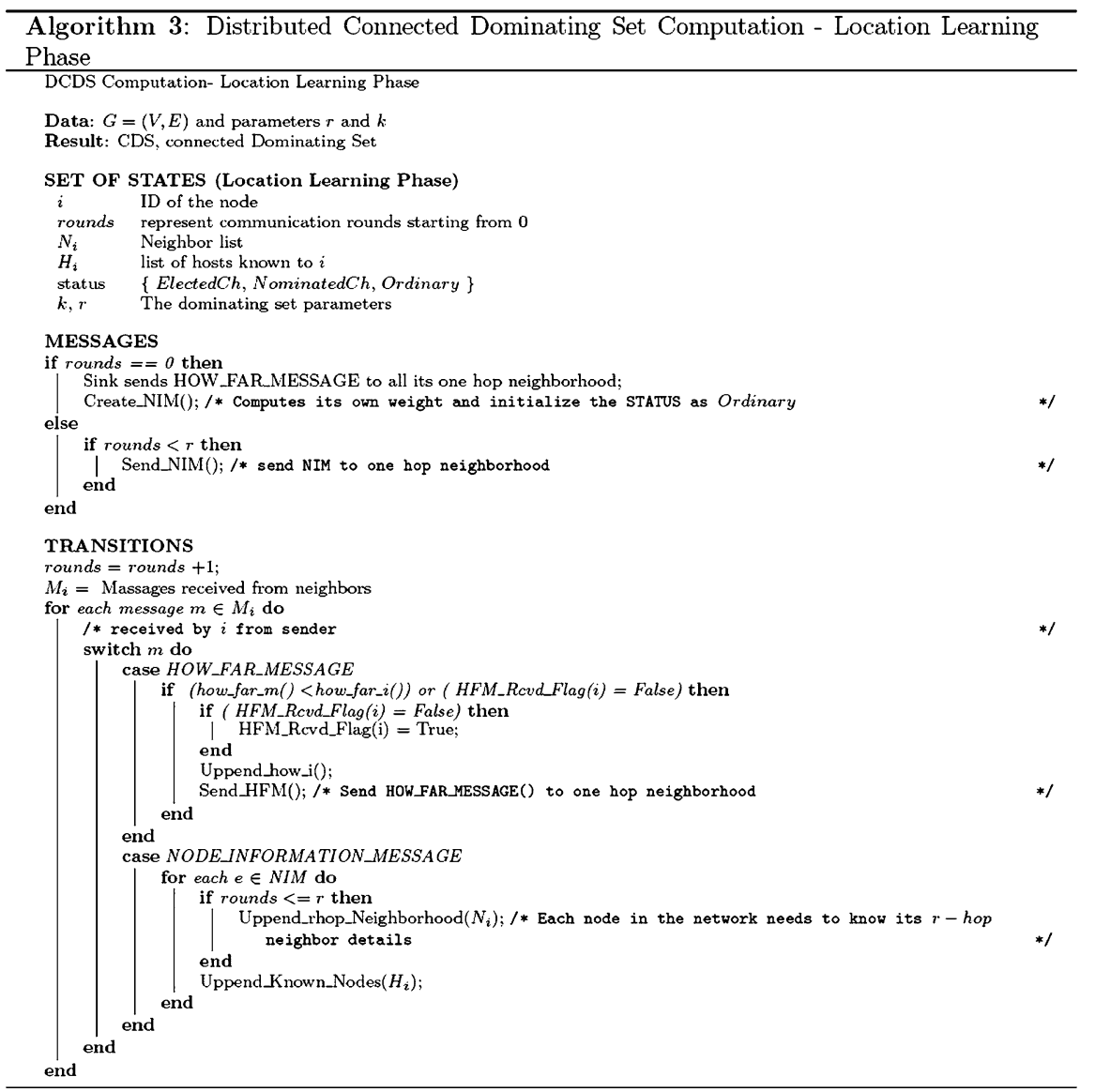


Fig. 6 CDS-computation (DCWACS)

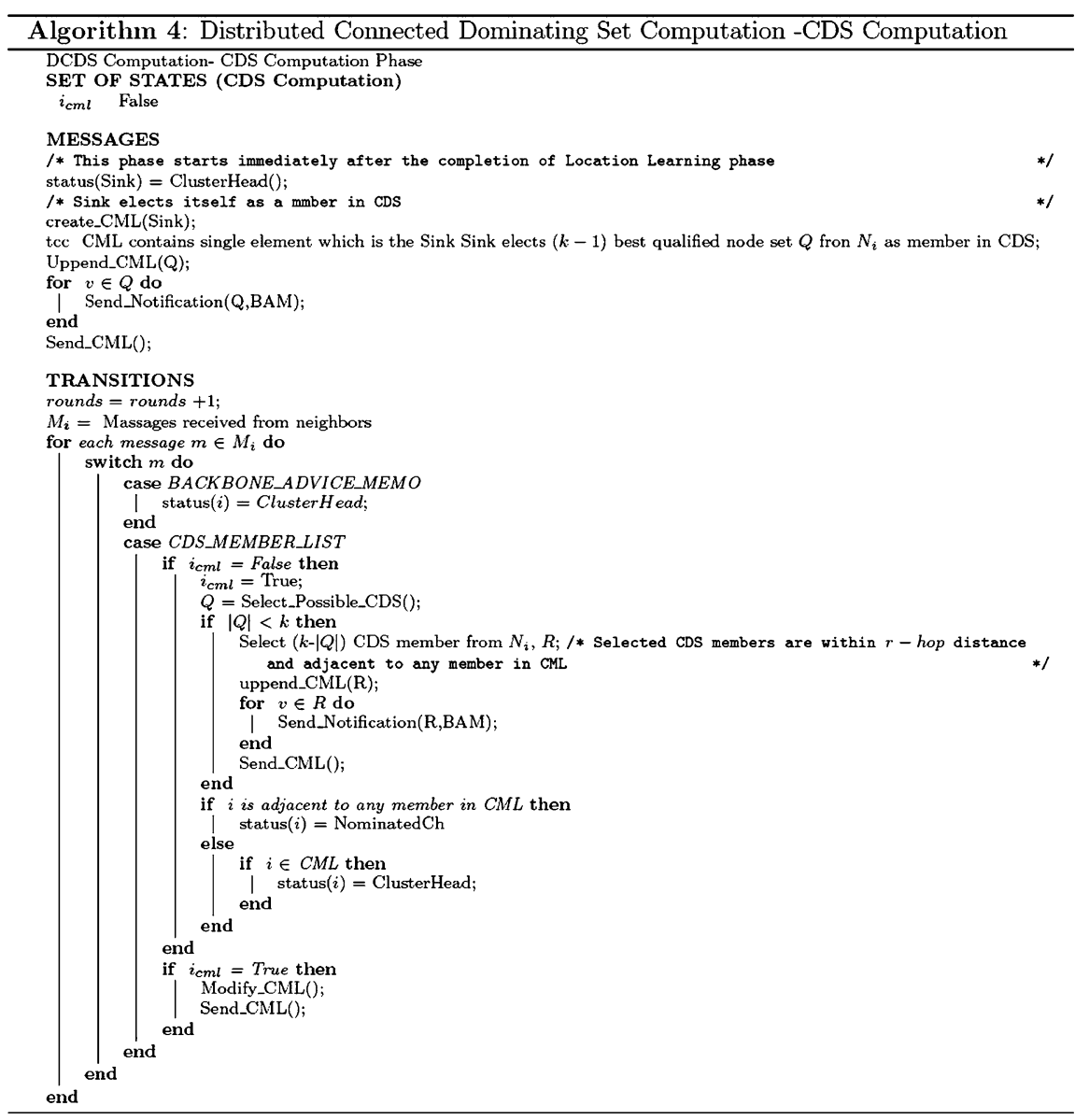

node computes its weight using (2) and sends the Node_Information_Message (NIM) to its $r$-hop neighborhood. In each round, the nodes broadcast the list of available NIM of nodes in the $r$-hop distance. After $r$ rounds, every node in the network learns about its $r$-hop neighborhood. This phase also computes the distance of each node from the Sink. The Sink initiates this phase by sending a How_Far_Message (HFM) to all its neighbors. On receiving $H F M$, each node updates the Distance_From_Sink (DIFS) field (which is zero for the initial message from the Sink) and sends this to all its neighbors. If a node gets more than one $H F M$, the message with the least value of DIFS triggers. Every node in the network learns its distance from the Sink in finite amount of time. Thus, at the end of Phase I, each node learns its distance from the Sink and also learns the details of its $r$-hop neighbors.

\subsubsection{CDS-computation}

This phase also starts from the Sink. The Sink elects itself as a member in the CDS and elects the $(k-1)$ best qualified (based on the degree, residual battery power, and transmission range) immediate neighbor nodes as members of the CDS. Ties are broken choosing the smallest distance. The CDS_MemberList $(C M L)$ message containing the list of elected members is send to its neighbors and the Backbone_Advise_Memo $(B A M)$ to the $(k-1)$ other elected neighbors to notify its selection. Upon receiving the $C M L$, each node $i$, selects the backbone elements either from this list or it elects a new member which is adjacent to any one of the members in the CML. This should be within the $r$-hop distance from $i$. This is done only once, immediately after receiving the first $C M L$ message. A node, after electing its backbone elements appends the $C M L$ by adding its elected members to the existing list and transmits the new message to its neighbors. New elections, if any, is intimated through the $B A M$. The CDS computation continues until all nodes in the network have posted their elections. Appropriate techniques are used to eliminate the duplicate messages. The algorithm for CDS-Computation is as shown in Fig. 6.

\subsubsection{Example}

Figure 3 shows the distributed $(2,2)-C D S$ for the typical sensor network shown in Fig. 1. To adhere to the property of sensor networks, we assume that the Sink has the highest weight in terms of battery power, transmission range, and degree, and is elected as the first element in the CDS. The CDS computation stems from the Sink. As shown in 
Fig. $3 \mathrm{~A}$, the node $\mathrm{G}$ has the highest weight and is elected as the Sink. After $r$ rounds, all nodes in the network are familiar with their $r$-hop neighborhood. Node G elects $(k-1)$ most qualified nodes as members in CDS. Since the $k$ value is 2 , node $\mathrm{G}$ elects the best qualified adjacent node $\mathrm{F}$ as the next member in the CDS. Node G sends the $C M L$ list to all its immediate neighbors and the Notification to the node F. During the next round, node F changes its status to ClusterHead and all Sink neighbors perform their election process as in (Fig. 3 B). Thus, nodes E, J, K, and F elect G and $\mathrm{F}$ and forward the $C M L$ to its neighbors.

Nodes A, B, D, H, and I are at 2-hop distance away from the Sink. A elects $\mathrm{G}$ and $\mathrm{E}$, similarly $\mathrm{B}, \mathrm{D}$, and $\mathrm{H}$ elect $\mathrm{F}$ and $\mathrm{G}$, whereas I elects $\mathrm{G}$ and $\mathrm{J}$, as shown in Fig. $3 \mathrm{C}$. In sequence, the next round $C M L$ reaches to all nodes at 3-hop distance from the Sink and node $\mathrm{C}$ elects $\mathrm{E}$ and F. This ends the election as all nodes in the network are covered and completed their election processes. The elected backbone elements are $\mathrm{E}, \mathrm{F}, \mathrm{K}$, and $\mathrm{J}$.

\subsubsection{Analysis}

Theorem 2 The distributed CDS algorithm correctly computes the $(k, r)$-CDS for any connected graph $G$.

Proof At the end of the Location Learning phase any node $n_{i}$ in the connected graph knows its $r$-hop neighbors, $V_{r d}$ and the distance to the Sink.

Initialization: Initially, the CDS contains a single node, $n_{0}$, which is the Sink (a connected, covered subgraph with single node $n_{0}$ ).

Maintenance: The node $n_{0}$ selects the most qualified (with larger weight values) nodes in the $r$-hop distance and sends the list of elected members using the $C M L$ message. On receiving the $C M L$ message, each node starts its CDS member election. This process is done only once and is done immediately after receiving the first $C M L$ message.

The CDS Member election is based on the following rules:

(i) $k$ nodes with the highest weight values within the $r$-hop distance are elected.

(ii) Elected node is either a member in $C M L$ or adjacent to any one element in $C M L$.

Rule (i) preserves the redundancy whereas rule (ii) preserves the connectivity. In each round, zero or more number of elements are added to the CDS by nodes in incremental values of the distance from Sink.

Termination: The process repeats until all nodes in the network complete its election. The total number of elements in the network is finite and takes finite amount of time to propagate throughout the network. Hence, the programme terminates within a finite amount of time.

\section{Cluster formation and management}

The cluster formation procedure starts after building the backbone structure (common for both centralized and distributed algorithms). The backbone members periodically send the Cluster_Head_Advertisement (CHA) to the ordinary nodes in the network (each node gets at least $k$ such messages from $k$ different backbone members). A logical relationship pair (NodId, ChId) is established on receiving the first CHA message from ChId. Using $t$ continuous $\mathrm{CHA}$ messages from the dominating set belonging to (NodId, ChId), an ordinary node with NodId computes $L S T A B$ and the quality using (7). After computing the quality of all $r$-hop neighboring backbone elements, an ordinary node $i$ sends Node_Join_Req (NJ) to the most qualified member in the backbone. If the total number of nodes attached with that cluster head is below the maximum allowable number then the clusterhead accepts this request and sends $N J \_A C K$ message for confirmation. If the ordinary node does not receive any $N J \_A C K$ message within the stipulated time, it sends the $N J$ message to the next qualified backbone node within the $r$-hop distance. Thus, the clusters are formed with CDS-members as clusterheads. A CDS member without clustermembers acts as a gateway. To improve power efficiency, a clusterhead schedule activities in its cluster so that the nodes can switch to the low-power sleep mode. This scheduling is done in round-robin order.

A good clustering algorithm should preserve the network structure as much as possible even when the nodes are moving around and the topology is changing slowly. The clusterhead re-election and the frequent information exchange between the participating nodes result in high computation cost overhead. The proposed algorithms avoid excessive computation in cluster maintenance and the cluster structure be preserved as long as possible. A Cluster management mechanism takes care of the addition of new nodes in the network. The hello message of the new node and the periodic CHA message of the CDS member help the cluster head association of the new node.

When a cluster member loses its contact with the current clusterhead, it selects the next best CDS node from the $(k-1)$ choices as its clusterhead and sends the $N J$ message to its new clusterhead. The clusterhead can fail by low battery, by switch off or by fail. When its residual battery is below $20 \%$, a clusterhead informs this message to all cluster members so that they can get attached with the next best choice as clusterhead by sending the $N J$ message.

The $(k, r)$ - $D S$-based algorithm improves reliability, provides variable degree of clusterhead redundancy and avoids frequent clusterhead re-elections. This algorithm is flexible in the determination of clusterhead density. Suitable cluster maintenance mechanisms are used to preserve the network topology as stable as possible. Unbalanced clusters cause 
uneven energy consumption and shorten the network lifespan. The algorithms use techniques to create load balanced clusters.

\subsection{Load balancing}

The backbone nodes support its members with the radio resources and route messages for the other nodes belonging to other clusters. The load handled by a backbone node depends on the number of member nodes in its cluster. It is desirable to have uniform load distribution among the backbone members. It is not practical to share the load with a node whose load is already close to the average network load. We use the Load Balancing Factor (LBF) to quantitatively measure how well balanced the nodes are. The LBF is given as the inverse of the variance of the load of the nodes, which is computed as

$L B F=\frac{N}{\sum_{i}\left(L_{i}-\mu\right)^{2}}$

where $N$ is the total number of nodes in the network, $L_{i}$ is the load of node $i$ and $\mu$ is the average network load. The average network load is computed as

$\mu=\frac{1}{N} \sum_{i \in V} L_{i}$

It is difficult to maintain a perfectly load balanced network. The LBF value tends to infinity for a perfectly balanced network.

The proposed algorithm achieves load balancing by redistributing the load to different clusterheads based on its quality and by specifying an agreeable threshold on the number of nodes that a clusterhead can handle.

\section{Performance evaluation}

The performance of CCWACS and DCWACS are evaluated through simulation. The parameters used in the simulation are listed in Table 1. We use a customized simulator in MATLAB. The nodes are randomly placed over a terrain and move according to the Random Waypoint Model. Only the connected topologies are taken into account. An ideal MAC protocol with no collisions is assumed for the simulations as in [32]. As no known optimum solution for the $(k, r)-C D S$ problem in WSN applications is available, the centralized $(k, r)$-CDS is used as the upper bound in the case of control overhead and lower bound in the case of backbone elements. The centralized algorithm uses many control messages for topology learning and for the $(k, r)-C D S$ computation but it creates a backbone with optimal number of nodes. The metrics used for the performance evaluation are (i) Cardinality of the CDS, (ii) Load balancing Factor, (iii) Number of reaffiliations, (iv) Network lifespan, and (v) Power dissipation.

Many CDS-based protocols and algorithms have been proposed in literature. Most of these algorithms are aimed in the construction of Minimum Connected Dominating Set and they create $(1,1)-C D S$ (CDS) or MCDS. Wu and Li proposed both centralized and distributed algorithms for CDS construction. Wu and Li's algorithm computes $k$-connected, $m$-dominating set CDS which is closer to our approach than the other popular $(1,1)-C D S$ algorithms. So, we use Wu and Li's algorithm for the comparison purposes.

The $(k, r)$-CDS simplifies the routing by restricting the main routing tasks only to a small number of nodes. The small size $(k, r)$-CDS reduces the number of nodes in the routing related tasks. Figures 7 and 8 show the dependence of $r$ on the number of elements in the backbone structure in centralized and distributed algorithms. As expected, the CCWACS creates a backbone structure with smaller number of nodes than the DCWACS. This is because in CCWACS, a node in the backbone structure is added in such a way that it covers most of the neighbor nodes. Whereas, in DCWACS the elements are added through an election process with the knowledge of the $r$-hop neighborhood, and hence the subset is not minimal. It can be observed that the number of backbone elements increases linearly with the number of nodes. In CCWACS, when the bounded distance parameter $r$ is increased from 1 to 2 there is 16 to $26 \%$ reduction in the total number of elements. There is a reduction of 21 to $25 \%$ in the backbone structure elements when the value of $r$ is increased from 2 to 3. In DCWACS, there is about 10 to $47 \%$ reduction in the number of backbone elements on increasing the value of $r$ from 1 to 2 . The rate of reduction is less when $r$ is increased from 2 to 3 (10 to 19\%). The backbone elements are elected based on the node weight. As the radius of the election process increases, more nodes select the same set of nodes in their nomination as backbone nodes. Figures 9 and 10 show the comparison of the size of backbone structure

Table 1 CCWACS/DCWACS parameters

\begin{tabular}{ll}
\hline Parameter & Value \\
\hline Simulator & MAtlab 7.1 \\
Simulation tool & Prowler \\
Number of nodes & $50-1000$ \\
Network size & $100 \times 100 \mathrm{~m}^{2}, 500 \times 500 \mathrm{~m}^{2}$ \\
Transmission range & $10 \mathrm{~m}-1000 \mathrm{~m}$ \\
Max speed of node movement & $10 \mathrm{mtrs} / \mathrm{sec}$ \\
$W_{1}, W_{2}, W_{3}$ and $W_{4}$ & $0.5,0.3,0.1 \mathrm{and} 0.1$ \\
Mobility model & Random way point \\
$E_{\text {elec }}, R_{\text {elec }}$ & $50 \mathrm{~nJ} / \mathrm{bit}$ \\
$T_{\text {amp }}$ & $100 \mathrm{pJ} / \mathrm{bit} / \mathrm{m}^{2}$ \\
Simulation time & $200 \mathrm{sec}$ \\
\hline
\end{tabular}




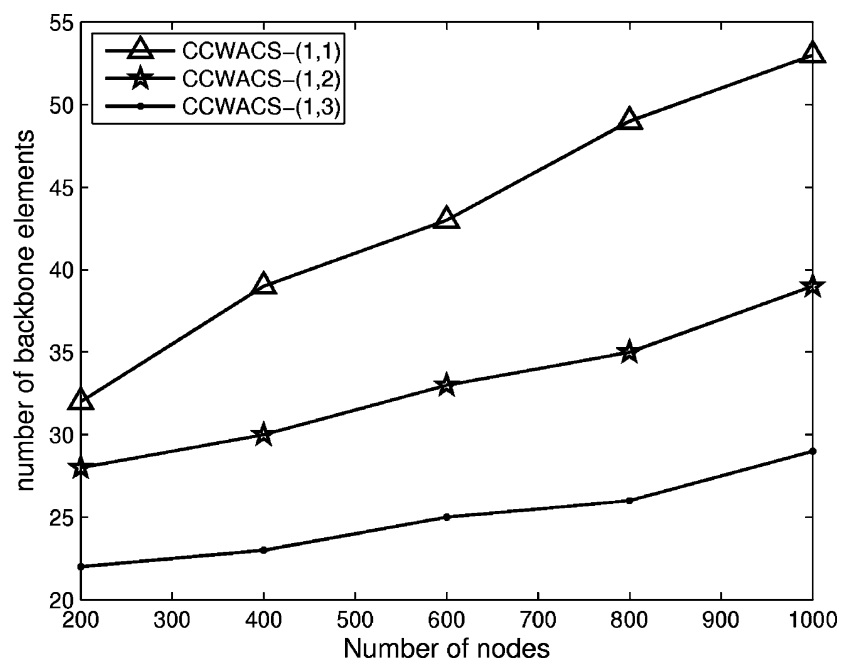

Fig. 7 Dependence of $r$ on the number of backbone elements in CCWACS

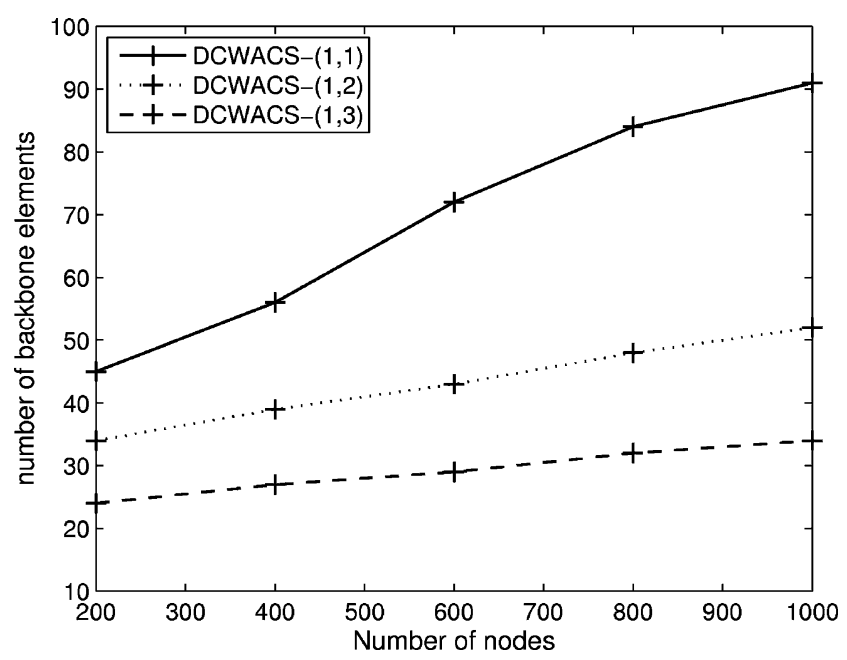

Fig. 8 Dependence of $r$ on the number of backbone elements in DCWACS

in CCWACS and DCWACS with Wu and Li, respectively. Both CCWACS and DCWACS create backbones with less number of nodes. The reduction in the number of backbone elements is from 20 to $48 \%$ in the case of CCWACS and 15 to $28 \%$ in DCWACS, in comparison with $\mathrm{Wu}$ and $\mathrm{Li}$.

The effectiveness of Load Balancing-heuristics in CCWACS and DCWACS is as shown in Figs. 11 and 12, respectively. A higher value of LBF signifies a better load distribution. The load balancing techniques used in these algorithms increase the LBF. As the existing algorithms do not support LBS, we do not have a bench mark for comparison purposes.

Figure 13 shows the re-affiliations per unit time in CCWACS with respect to the transmission range. For small values of the transmission range, more number of stable clusters are created, and hence re-affiliations are less. The

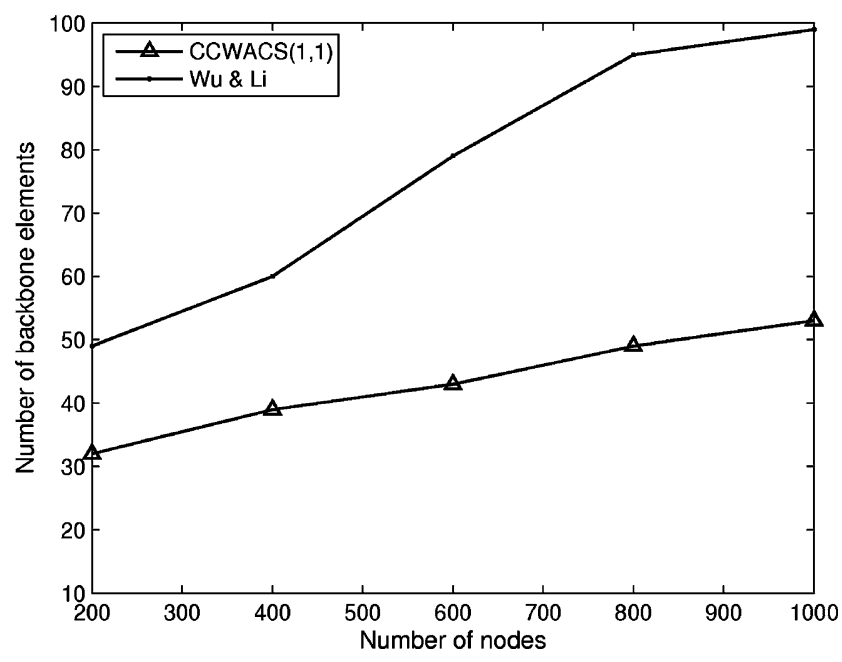

Fig. 9 Comparison of cardinality of backbone structure in CCWACS and $\mathrm{Wu} \& \mathrm{Li}$

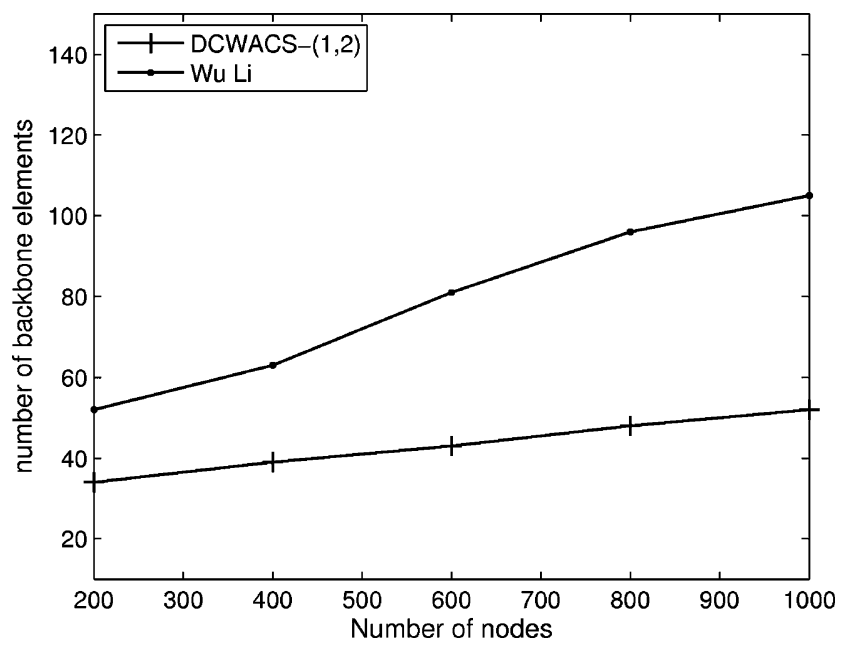

Fig. 10 Comparison of cardinality of backbone structure in DCWACS and $\mathrm{Wu} \& \mathrm{Li}$

number of re-affiliations increases as the transmission range increases. When the transmission range is about 40 meters, the number of re-affiliations takes the maximum value, and further increase in the transmission range leads to less number of re-affiliations. This is because the elected members in the backbone structure cover larger areas and the mobile nodes tend to stay within this large area. DCWACS also shows similar results. Figure 14 shows the comparison of the number of re-affiliations in DCWACS and Wu and Li. It is observed that the number of re-affiliations is much less in DCWACS than in Wu and Li. This is because each ordinary node in the network selects the most suitable node as its clusterhead to construct stable clusters.

In a direct communication protocol, each sensor node sends its data to the Sink directly. Nodes which are near to the Sink require less transmit power than the nodes located far away from the Sink. So, the nodes far away from the 


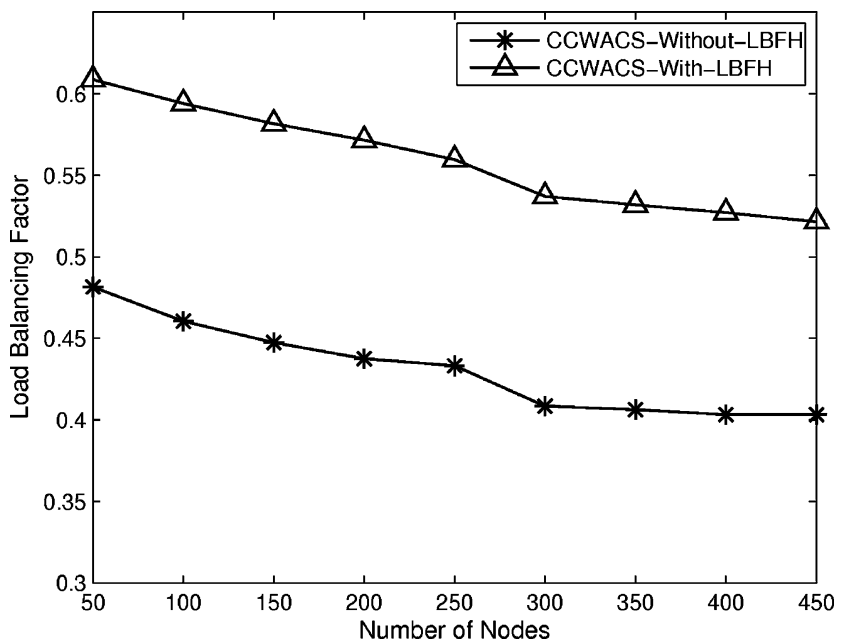

Fig. 11 Effectiveness of LBF-Heuristics in CCWACS

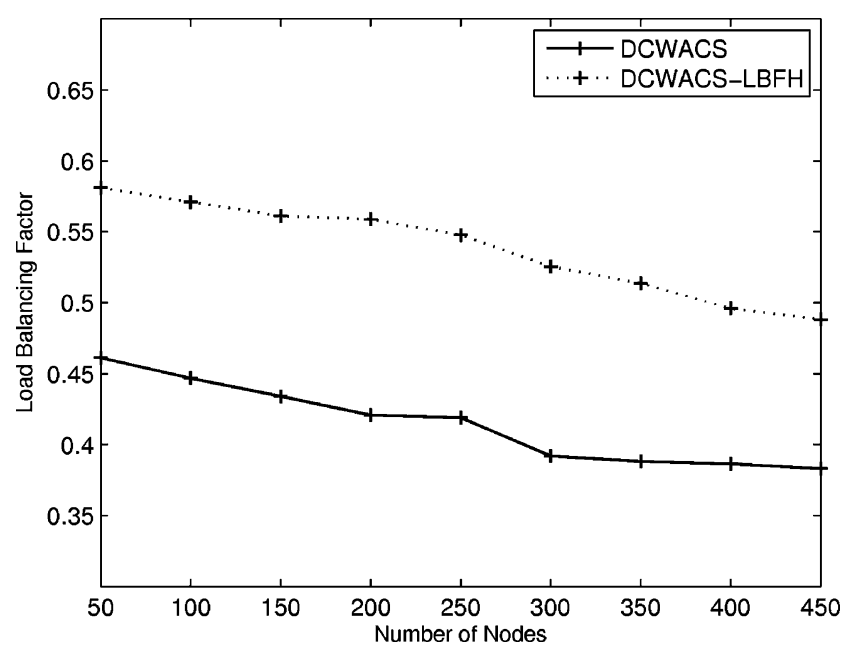

Fig. 12 Effectiveness of LBF-Heuristics in DCWACS

Sink quickly drain their battery power and drop out of the network. The Minimum-Energy Multi-hop routing (MTE) uses intermediate nodes for communicating with the Sink. Hence, in MTE routing, the nodes near to the Sink are used for routing a large amount of messages to the Sink and die earlier than the nodes far away from the Sink. In CCWACS and DCWACS also, the nodes in the B-structure can communicate either directly or through multi-hop to the Sink.

The lifetime of a network is the number of rounds completed without a single hole in the network or the number of rounds completed with at least a single active node remaining in the network. Table 2 shows the comparison of the first node death and the last node death for the different protocols. In CCWACS-M and DCWACS-M, the first node death is found earlier than in CCWACS-D and DCWACS-D, respectively. But the last node dies earlier in CCWACS-D and DCWACS-D than in the corresponding multi-hop transmissions. CCWACS and DCWACS (both Direct and Multi-hop)

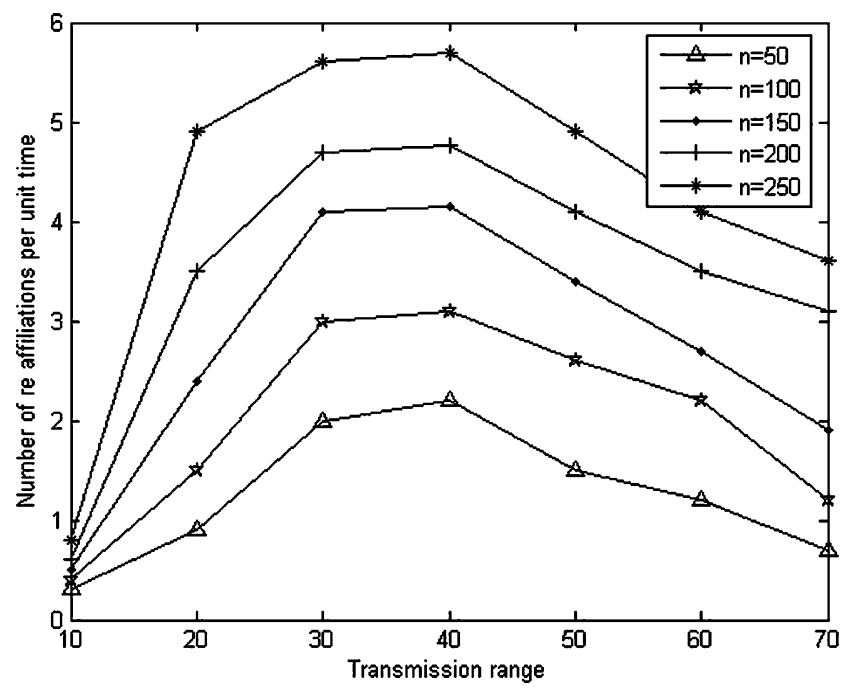

Fig. 13 Number of re-affiliations per unit time Vs Transmission range

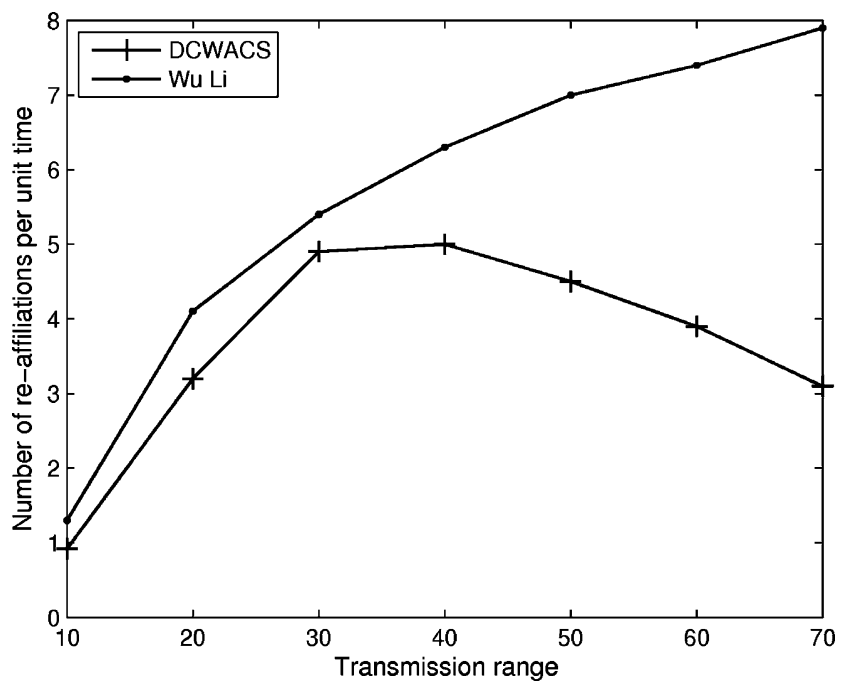

Fig. 14 Comparison of the number of re-affiliations in DCWACS and $\mathrm{Wu}$ and $\mathrm{Li}$

Table 2 Lifetimes of the protocols (in number of rounds)

\begin{tabular}{lcl}
\hline Protocol & First node dies (rounds) & Last node dies (rounds) \\
\hline Direct & 45 & 115 \\
MTE & 8 & 212 \\
CCWACS-D & 63 & 418 \\
CCWACS-M & 55 & 479 \\
DCWACS-D & 73 & 465 \\
DCWACS-M & 64 & 514 \\
Wu and Li & 51 & 341 \\
\hline
\end{tabular}

have longer lifetime than the Direct, MTE, and $\mathrm{Wu}$ and $\mathrm{Li}$ protocols. This is because the nodes in the B-structure are 


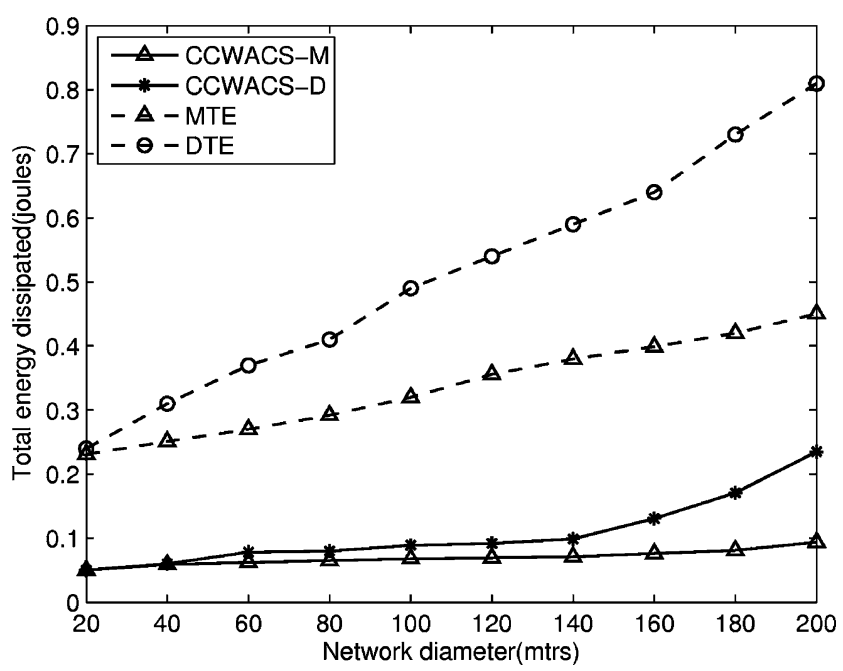

Fig. 15 Total power dissipated using Direct, MTE, CCWACS-D, and CCWACS-M for a random network

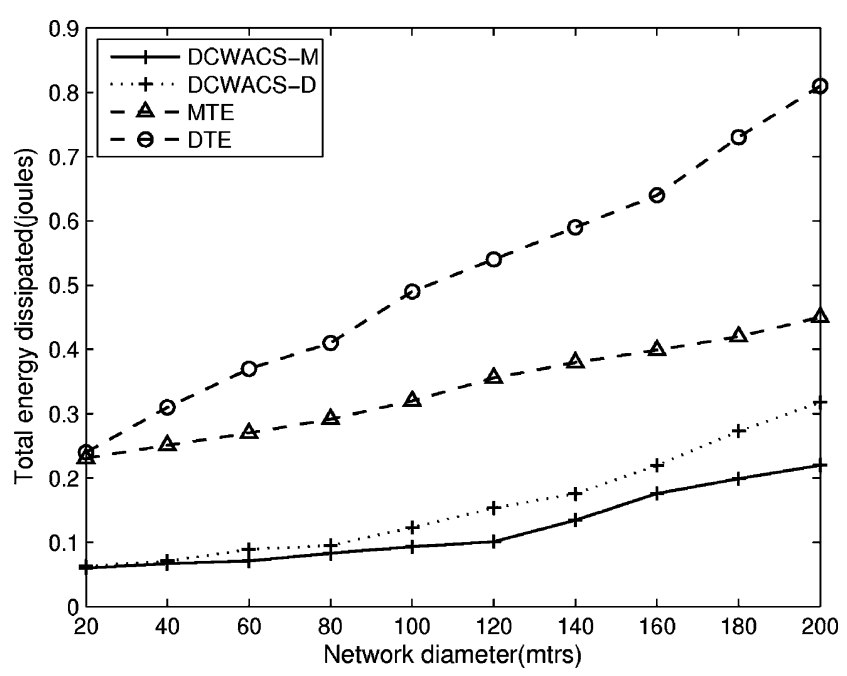

Fig. 16 Total power dissipated using Direct, MTE, DCWACS-D, and DCWACS-M for a random network

selected based on its residual battery power, node degree, transmission range, and mobility.

Figures 15 and 16 show the total energy dissipated for the scenario in which each node has a 2500 bit packet to send to the Sink. The random network with $E_{\text {elec }}, R_{\text {elec }}$ and $T_{\text {amp }}$ as shown in Table 1 is used. It is observed that CCWACS$\mathrm{M}$ and DCWACS-M save an average of $70 \%$ and $58 \%$, respectively, of the total energy dissipated in comparison with MTE. Similarly, CCWACS-D and DCWACS-D save an average of $65 \%$ and $60 \%$, respectively, of the of total energy dissipated in comparison with the Direct protocol.

\section{Conclusions}

In this paper, we have presented connected, dominating setbased, weighted, and adaptive clustering algorithms (Cen- tralized and Distributed) for ad hoc sensor networks. The elements in the backbone structure are selected based on the $(k, r)-C D S$. The Centralized-algorithm computes the dominating set elements progressively, taking the most suitable sensor node based on its weight, as member in the dominating set. The Distributed-algorithm uses a distributed method for the selection of the CDS members. The $(k, r)-C D S$ provides clusterhead redundancy in multi-hop scenarios.

The proposed algorithms allow variable diameter clusters and clusterhead redundancy for scalability. These algorithms optimize the number of clusterheads and provide load balancing. They limit the number of re-affiliations of nodes making the WSN more stable. They minimize the total power dissipation of the network there by maximizing the lifetime of the network.

In many sensor network applications, there may be a Sink with continuous power supply and other resources. The CCWACS does not scale well due to the extra control overhead for learning the network topology and so the centralized-algorithm is more suitable for smaller and deterministic topologies. Though the CCWACS requires additional control messages for learning the network topology but its performance is good in terms of the number of dominating sets in the backbone structure. The DCWACS is adaptive and creates stable, reliable, and load balanced clusters with less control overhead and longer lifespan. DCWACS uses the distributed election mechanism for the selection of the CDS members (without knowing the entire topology and with less number of control messages), and hence DCWACS is suitable for WSN with large number of nodes.

Incorporating security and data aggregation capabilities to the protocols are topics suggested for further research.

\section{References}

1. Agre J, Clare L (2000) An integrated architecture for cooperative sensing networks. Computer 33(5):106-108

2. Akkaya K, Younis M (2005) A survey on routing protocols for wireless sensor networks. Ad Hoc Netw 3(3):325-349

3. Akyildiz IF, Su W, Sankaraubramaniam Y, Cayirci E (2002) A survey on sensor networks. IEEE Commun Mag

4. Akylidiz IF, Su W, Sankarasubramanian Y, Cayirci E (2002) Wireless sensor network: A survey on sensor network. IEEE Commun Mag 40(8):102-114

5. Anitha VS, Sebastian MP (2009) Scenario-based cluster formationand management in mobile ad hoc networks. Int J Mobile Comput Multimedia Commun 1(1):1-15 (IGI Journal)

6. Anitha VS, Sebastian MP (2009) Scenario-based diameterbounded algorithm for cluster creation and management in mobile ad hoc networks. In: 13th IEEE/ACM international symposium on distributed simulation and real time applications, pp 97-104

7. Blum J, Ding M, Thaeler A, Cheng X (2004) Connected dominating set in sensor networks and MANETs. In: Du D-Z, Pardalos P (eds) Handbook of combinatorial optimization. Kluwer Academic, Amsterdam

8. Bonnet P, Gehrke J, Seshadri P (2000) Querying the physical world. IEEE Pers Commun 7(5):10-15 
9. Bulusu N, Estrin D, Girod L, Heidemann J (2001) Scalable coordination for wireless sensor networks: self-configuring localization systems. In: International symposium on communication theory and applications (ISCTA 2001). Ambleside, UK, 2001

10. Buratti C, Conti A, Dardari D, Verdone R (2009) An overview on wireless sensor networks technology and evolution. Sensors 9(9):6869-6896

11. Chatterjee M, Das SK, Turgut D (2002) Wca: a weighted clustering algorithm for mobile ad hoc networks. Clust Comput 5(1):193-204

12. Chen Y, Lieshman AL (2002) Approximating minimum size weakly connected dominating sets for clustering mobile ad hoc networks. In: MobiHoc. ACM Press, New York, pp 165-172

13. Das B, Bhargavan V (1997) Routing in ad hoc networks using minimum connected dominating set. In: ICE, pp 371-380

14. Das B, Sivakumar E, Bhargavan V (1997) Routing in ad hoc networks using a virtual backbone. In: Proceedings for the 6th international conference on computer communications and networks (IC3N'97), Las Vegas, NV, USA, 1997, pp 22-25

15. Das B, Sivakumar R, Bharghavan V (1997) Routing in ad-hoc networks using a spine. In: Proc of international conference on computers and communications networks, ICCCN, Las Vegas, 1997

16. Ding P, Holliday J, Celek A (2005) Distributed energy efficient hierarchical clustering for wireless sensor networks. In: Proc of the IEEE international conference on distributed computing in sensor systems

17. Friis: (1946) A note on simple transmission formula. In: Proceedings of IRE, pp 254-256

18. Garey M, Johnson D (1978) Computers and intractability: a guide to NP-completeness

19. Guha S, Khuller S (1998) Approximation algorithms for connected dominating sets. Algorithmica 20:374-387

20. Halweil B (2001) Study finds modern farming is costly. World Watch 14(1):9-10

21. Heinzelman W, Chandrakasan A, Balakrishnan H (2002) An application-specific protocol architecture for wireless microsensor networks. IEEE Trans Wirel Commun 1(4):660-670

22. Johnson P, Andrews DC (1996) Remote continuous physiological monitoring in the home. J Telemed Telecare 2(2):107-113

23. Kahn J, Katz R, Pister K (1999) Next century challenges: mobile networking for smart dust. In: Proceedings of the ACM MobiCom'99. Washington, USA, 1999, pp 271-278

24. Li J, Andrew LL, Foh CH, Zukerman M, Chen HH (2009) Connectivity, coverage and placement in wireless sensor networks. Sensors
25. Lindsey S, Raghavendra CS (2002) Pegasis: power efficient gathering in sensor information systems. In: Proc of IEEE aerospace conference. IEEE

26. Noury N, Herve T, Rialle V, Virone G, Mercier E, Morey G, Moro A, Porcheron T (2000) Monitoring behavior in home using a smart fall sensor. In: IEEE-EMBS special topic conference on microtechnologies in medicine and biology, pp 607-610

27. Paruchuri V, Durresi A, Durresi M, Barolli L (2005) Routing through back bone structures in sensor networks. In: Proceedings ICPADS, ICPADS, Japan, 2005, pp 397-401

28. Rabaey J, Ammer M, da Silva J Jr, Patel D, Roundy S (2000) Picoradio supports ad hoc ultra-low power wireless networking. IEEE Comput Mag

29. Ryl DS, Stojmenovic I, Wu J (2005) Energy-efficient backbone construction, broadcasting, and area coverage in sensor networks. Handbook of sensor networks. Wiley, New York

30. Sinha P, Sivakumar R, Bharghavan V (2001) Enhancing ad hoc routing with dynamic virtual infrastructures. In: 20th annual joint conference of the IEEE computer and communications societies, vol 3

31. Stojmenovic I, Wu J (2004) Broadcasting and activity scheduling in AD HOC networks. In: Basagni S, Conti M, Giordano S, Stojmenovic I (eds) Mobile ad hoc networking. IEEE, New York

32. Wan PJ, Alzoubi KM, Frieder O (2004) Distributed construction of connected dominating set in wireless ad hoc networks. Mobile Netw Appl 9:141-149

33. Warneke B, Liebowitz B, Pister K (2001) Smart dust: communicating with a cubic-millimeter computer. IEEE Computer, New York

34. Wu J, Li H On calculating connected dominating set for efficient routing in ad hoc wireless networks. In: Proc of proceedings of the 3rd ACM international workshop on discrete algorithms and methods for mobile computing and communications, pp. 7-14. ACM, New York

35. Wu Y, Li Y (2008) Construction algorithms for $k$-connected $m$-dominating sets in wireless sensor networks. In: Mobihoc 2008

36. Wu Y, Wang F, Thai MT, Li Y (2007) Constructing $k$-connected $m$-dominating sets in wireless sensor networks. In: Military communications conference. MILCOM, Orlando, pp 29-31

37. Younis O, Fahmy S (2004) Heed: A hybrid energy-efficient distributed clustering approach for ad hoc sensor networks. IEEE Trans Mob Comput 3(4):366-379 